Surveys in Differential Geometry XVII

\title{
Topics on Geometric Analysis
}

\author{
Shing-Tung Yau
}

I recall that about twenty years ago, Peter Li told me that the National Science Foundation has a program called geometric analysis. I was curious about what that was until Peter explained to me. I believe this is a real contribution of NSF to mathematics, on top of the funding that we have enjoyed all these years.

Just like any subject in mathematics, the roots of geometric analysis dates to ancient times and also to the not so ancient contributions of many modern mathematicians. And as many scientists like to say, we stood on the shoulders of giants, who laid down the basic tools and concepts and made important progress. But I believe only starting in the 1970s, did we see a systematic development based on analysis and nonlinear differential equations to solve important problems in geometry and topology. This development is vigorous and it has had strong feedback to the development of differential equations and analysis in general.

I remember that as a graduate student, I read a great deal of Russian literature on surface theory based on the Alexandrov school and works of Pogorelov. I also noticed the great works of Morrey, Nash, Nirenberg, de Giorgi and others. But they were more interested in analysis than geometry. Problems in geometry are good testing grounds for developments of partial differential equations. This is still so. But we started to develop into other directions.

When I entered the subject, it seemed to me that Riemannian geometry could benefit from the introduction of techniques beyond the geodesic methods which were almost exclusively used at that time. Indeed, the great power of the Atiyah-Singer Index Theorem had shown prevailing influence in the subject of geometry, topology and algebraic geometry already. On the other direction, the deep insights offered by integration by part and

This article is based on the C.-C. Hsiung Lecture that the author gave on the International Symposium on Geometry and Topology in Memory of C.-C Hsiung at Lehigh University on May 28, 2010.

(C)2012 International Press 
by the maximum principle in partial differential equations work like magic, once the right quantity is found.

I was convinced that there are much for geometers to learn from analysis and partial differential equations. With many friends with great strengths in both analysis and geometry, we started to look into various subjects such as minimal submanifolds, harmonic maps, Monge-Ampère equations, and Ricci flows.

I shall select a few of these topics and discuss their prospects. And I should stress that the subject matter of geometric analysis covers much more than the topics that I am going to discuss here.

Let me quote my good friend Hamilton: "The subject has just begun."

It will be a pity if young bright geometers are misled by ignorant comments that geometric analysis is dead. If we want to construct an interesting and deep geometric structure over a manifold that is not known to us in any concrete manner, differential equations seem to be the only way that I know of. This was indeed the guiding principle to prove the Poincaré conjecture or the geometrization conjecture using Ricci flow.

\section{Hamilton's Theory of Ricci Flow}

I recall that I met Hamilton in Cornell in 1979, who told me that he was working on the Ricci flow. At the same period, I was talking with Rick Schoen then in a similar direction of adapting the ideas of harmonic maps to study deformation of metrics. It was indeed a very natural concept. I may also say that the physicist Friedan also had came up with the equation in quantum gravity as a renormalization group flow. I recall that many analytic approaches to the solution of the Poincaré conjecture were proposed by geometers. But we all know that practically all of these other approaches led to ashes.

Friedan never really thought in terms of using the flow for understanding the structure of geometry or topology per say. The later claims of how string theory or physics led to the break through in Ricci flow, is just a myth. It did not really occur in that way.

In any case, the key to the development of Ricci flow is of course what we can do with such equations. I thought that Hamilton was just toying around with superficial claims until in 1980, he called me explaining what he could do with the Ricci flow for three-manifolds with positive Ricci curvature [26]. Immediately I realized that to be a major breakthrough for the whole subject of flows for geometric structures.

I had three of my graduate students to work on it. I instructed Bando [1] to work on Kähler-Ricci flow. He gave the first major result there: that positivity of bisectional curvature is preserved in complex three dimensions. Then I told Mok [51] to generalize it, which he did.

I told Cao and Chow $[\mathbf{8}]$ to use the flow to reprove the Frankel conjecture. Unfortunately, despite many attempts by my later students who claimed to 
have success, the problem is still not solved - basically the flow proof of the Frankel conjecture still depends on the argument that Siu and I [63] used in 1979 .

The news media has created an image that Hamilton did not do anything since the paper in 1983 until the work of Perelman $[\mathbf{5 5}, \mathbf{5 6}, \mathbf{5 7}]$. This is far from true. In fact, great progress was made in those 20 years. Creative new techniques were introduced by Hamilton, most of which revolutionized the subject of geometry. The deep understanding of Ricci soliton to understand singularities was made by Hamilton [27]. The profound technology of metric surgery was introduced by him [29]. He was able to give a deep and precise description of the metric when it is close enough to the singularity [28]. This includes statements on how a metric looks like when it starts to break up or acquire a singularity. In particular, it includes a true understanding of the Mostow Rigidity Theorem when the metric is only close to the hyperbolic space in a rough sense [30].

Hamilton [27] introduced a great deal of analysis beyond the classical study of partial differential equations. He managed to generalize the work of Li-Yau $[\mathbf{4 3}]$ on understanding the behavior of the heat equation. He adapted the argument to the Ricci flow in an ingenious manner. Note that Perelman [55] proved a noncollapsing estimate by proving Li-Yau's inequality on backward solutions to the adjoint heat equation, which he used to rule out the cigar singularity, which was anticipated by Hamilton. The generalization by Hamilton of the Li-Yau estimate is fundamental to several important points for understanding the singularities of the Ricci flow. All of these works are employed in the study of the Poincaré conjecture and the geometrization conjecture.

Let me say that the great prowess of Ricci flow goes way beyond the Poincaré conjecture, despite what some so-called experts said in the news media, that the method of the proof has no other future directions. On the contrary, I like to mention a few examples here.

We saw the success of Brendle-Schoen [3] using Ricci flow to prove the quarter pinching conjecture of the differentiable sphere theorem: a conjecture that seems to be far beyond what classical comparison theorems for geodesics can achieve. One sees that new techniques in partial differential equations are created and new problems are waiting to be solved.

There are natural open problems in metric geometry that should be able to be solved by Ricci flow. I would like to encourage our young geometric analysts to spend time on them:

A. A flow proof of the Gromoll-Meyer-Cheeger-Perelman $[\mathbf{1 1}, \mathbf{5 4}]$ soul theorem of complete open manifolds with non-negative curvature. Perhaps a flow argument can also be used to prove the converse: that the total space of any vector bundle over a compact manifold with positive curvature admits a complete metric with non-negative curvature? 
B. A proof of Gromov's theorem [24] of a bound on the Betti numbers of manifolds with positive sectional curvature depending only on dimension, settling the conjecture that the torus is the worst case. It is known that this follows from the statement that the Betti numbers of the loop space of the manifold grow at most polynomially. Perhaps it is instructive to compare the approach of Micallef-Moore [50] on the proof of the topological pinching theorem by studying the index of the Sacks-Uhlenbeck spheres. Can one use minimal surface techniques to settle this problem?

Of course, one would like to go beyond what is known in the area of metric geometry. For example, the following topics may be interesting:

Prove that there is only a finite number of manifolds with positive sectional curvature for each dimension when the dimension are greater than a large number. The number can be 25 . In fact, would all these be compact symmetric spaces? If so, Ricci flow should be very useful as we are now trying to flow it to some canonical models.

The problem of understanding Hamilton's flow on manifolds of negative curvature has not been explored as much as it can be. Perhaps some modifications are needed. The structure of the fundamental group of such manifold needs to be understood. For example:

C. Thurston made the conjecture that each hyperbolic three dimensional manifold is covered by another compact manifold which admits an embedded incompressible surface with genus $g>1$. Can one prove this statement based on analysis?

D. Given a compact manifold with negative curvature, can one find a "canonical" embedding of its universal cover into an open subset of some compact manifold with positive Ricci curvature so that all the deck transformations can be extended to be homeomorphisms of the larger manifold which may satisfy additional properties such as quasi-conformality? Hopefully, this can be simpler if the manifold is Kähler and in that case, we like to have everything to be holomorphic. The boundary of the open set could be complicated, but can be understood by looking at the set of the domain of discontinuity of the group.

E. For most structures that are canonically defined by the metric, it should be compatible with the Ricci flow. In those cases, the Ricci flow should give powerful information.

That was one of the reasons that I asked my former students to work on Kähler-Ricci flow. Unfortunately, besides the important advances made by Donaldson [15], the progress has been slow, since the thesis of Cao [5]. The most recent estimates of Perelman [55] should shed more light on how to proceed. The major question is how to understand the question of stability of the complex structure 
through the flow. After all, the conjecture that I [70] made on the equivalence of manifold stability with the Einstein condition should certainly show up in the study of the limit of the flow. One should study the limit of the Kähler-Ricci flow carefully to find the right definition of stability of Fano manifolds.

F. Kähler-Ricci flow has been used to help answer my conjecture [69] that a complete non-compact Kähler manifold with positive bisectional curvature is biholomorphic to $\mathbb{C}^{n}$. So far the best result on this is by Chau-Tam [10], who proved the conjecture in the case of bounded curvature and maximal volume growth. The work of Chau-Tam and others that use Käher-Ricci flow to address my conjecture make use of the important Li-Yau-Hamilton inequality, which was proved to hold for Kähler-Ricci flow by Cao [6, 7]. It should be possible to complete the program in the near future.

G. For manifolds with non-positive Chern classes, the problem of metric singularities near base points of the canonical map is very important for applications to algebraic geometry. The same question applies to non-compact Kähler-Einstein manifolds with nonpositive Chern class. One would like to study the structure of the points that compactify the manifold, and we hope to find the ansatz or structure of the metric at infinity. We also like to be able to calculate the residue of Chern classes at the singular points or at infinity. Hence a good description of the metric should include such information. The first paper in this direction was described by me in $1978[\mathbf{7 1}, \mathbf{7 2}, \mathbf{7 3}, \mathbf{7 4}, \mathbf{7 5}]$, where I proved that for algebraic manifold M with a divisor D, that M-D admits a canonical KählerEinstein metric if $K+D>0$. The singularity of the metric near D is well understood.

Once the structure of those singular metrics are understood, the application to algebraic geometry should follow easily. This should include the minimal model theory, the abundance conjecture and the structure of the moduli space of algebraic manifolds. In my original paper on the Calabi conjecture, I made the first attempt to understand the singularity of metrics. But there was not enough knowledge of algebraic geometry to help then and I hope our knowledge is matured enough to tackle such problems now.

H. The full theory of Ricci flow with boundary is not well developed yet. It will be nice to see how to deform such manifolds to Einstein manifolds whose boundary has constant mean curvature. We also need to develop a theory of Ricci flow for complete non-compact manifolds with no upper bound on curvature. In particular, prove the theorem of Schoen-Yau [60] that every complete non-compact three dimensional manifolds with positive Ricci curvature is diffeomorphic to Euclidean space. 
I. Many years ago, I [70] proposed to study the groups of exotic spheres in the following way: An exotic spheres admit metrics with positive scalar curvature iff it bounds a spin manifold. An exotic spheres admit metrics with positive Ricci curvature iff it bounds a parallelizable manifold. The first statement was proved by the work of Stolz [64] based on the surgery result of Schoen-Yau [59] and Gromov-Lawson [25]. The second assertion can be refined by replacing parallelizable manifolds with manifolds where some of their Pontryagin classes are zero, while we only require some of the eigenvalue of the Ricci curvature to be positive. Perhaps we can achieve this by flows on compact manifolds with boundary.

J. Can one find a reasonable canonical bounding manifold, similar to those appear in AdS/CFT [68] theory where we require the bounding manifold to admit an Einstein metric that is asymptotic to hyperbolic space near the boundary?

K. Can one characterize those exotic spheres that admit metrics with positive sectional curvature? Should they admit a fiber structure where both fiber and base admit metrics with positive curvature so that the fiber bounds a manifold with positive curvature in a smooth fashion?

L. It is well known that an evolution equation very close to Hamilton's Ricci flow is the mean curvature flow, where several people, starting from Gage [19], Hamilton [20], Grayson [23], and Huisken $[\mathbf{3 4}, \mathbf{3 5}]$, made important contributions. It remains to be seen how to use the mean curvature flow, or other submanifold flows, to handle the famous problem of the Schoenflies conjecture [17], that any smoothly embedded three-sphere in $\mathbb{R}^{4}$ bounds a four-ball.

\section{Manifolds with Special Holonomy Groups}

Can flows generate manifolds with $G_{2}$ or $\operatorname{Spin}(7)$ holonomy groups? Compact manifolds of these types were constructed by Joyce $[\mathbf{3 7}, \mathbf{3 8}, \mathbf{3 9}]$. But unfortunately, Joyce's manifolds are based on singular perturbation methods, and it is difficult to use the method to study the global moduli problem, which is important for the M-theory linked to the manifold. It will be great to find a flow or other global elliptic method to construct such manifolds.

There are some obvious obstructions for a manifold to admit $G_{2}$ structures, in terms of nontriviality of the third cohomology and positive definiteness of the quadratic form associated to the first Pontryagin form, as can be found in the book of Joyce [40]. To the best of my knowledge, these are the only known obstructions. Can one prove it?

Many construction of such manifolds are related to constructions of Calabi-Yau manifolds, and there are proposals to relate them to a pair of Calabi-Yau manifold plus a special lagrangian cycle in this manifold. It was

proposed that some total space of a circle fiber space over the manifold 
degenerate along the cycle may do the job. Such constructions work better for non-compact manifolds and the technology is far from mature.

Special holonomy manifolds play an important chapter in differential geometry and physics. However, there are other manifolds of interest that do not have special holonomy group. The most notable of such manifolds are Einstein manifolds. Major construction of such manifolds either come from Kähler geometry, from reduction of dimension by group actions or from the Wick rotation from Lorentzian Einstein manifolds [21]. It will be interesting to see how general such constructions are.

This is especially true for the Wick rotation construction: it is possible to start out from a singular Lorentzian manifold and obtain a non-singular Einstein manifold. Under what conditions can we reverse this procedure to produce interesting solutions to the Einstein equation which have physical interest?

When we study cone singularities for manifolds with a special holonomy group, we can find new structures, such as Sasaki-Einstein manifolds [4] or nearly-Kähler manifolds [22]. They themselves do not have special holonomy group and yet they are constructed from such manifolds by reduction of dimension. If we look at the category of all manifolds generated by manifolds with special holonomy group by doing reduction of this sort or manifolds with a foliation whose leaves are given by special holonomy group, how big a class of manifolds can we generate? How do we characterize them?

\section{Four Manifolds}

A very important problem regarding structures on manifolds relate to four dimensional manifolds where few structures are known. But there is an important one, besides the space of Einstein metrics. These are the self-dual or anti-self-dual metrics. They are naturally defined for four-dimensional manifolds and their existence says a lot about the manifolds. For one thing, the twistor space of anti-self-dual manifolds admits an integrable complex structure and the anti-self-dual bundles lift to holomorphic bundles. It therefore has a natural connection to the theme of complex geometry.

Hitchin $[\mathbf{3 1}]$ showed that the twistor space is not Kähler except for a couple of obvious examples. However, they can have a lot of meromorphic functions and naturally the function field gives us natural invariants of the manifolds. There is also the concept of Kodaira dimension of the complex manifold that may give some understanding for the anti-self dual metrics.

While there are many constructions of self-dual manifolds, especially the spectacular works of Taubes on the existence of such metrics after stabilization by connected sums with complex projective plane, the general existence theorem is still not known.

But in order to understand such metrics, we need to be able to give direct proof of existence without using singular perturbation method (Perhaps one can use flows or elliptic techniques). We need to understand the 
global moduli space of such metrics. In particular, do they have an infinite number of components once we fix the diffeomorphic type of the manifold. This is of course, very relevant to the question of the topology of four dimensional manifolds.

Similar questions can be addressed for Einstein metrics. Our understanding on Einstein manifolds is also rather poor. We are not even sure whether there can be more than two distinct Einstein metrics on the four sphere.

\section{Six Dimensional Manifolds}

Six dimensional geometries motived by string theory have pointed to new directions to look for geometric structures. The first important question is to give a topological classification of all Calabi-Yau manifolds. But it is also interesting to study the non-Kähler complex manifolds. I believed that in six dimensions, any almost complex manifold admits an integrable complex structure. If my conjecture is true, the problem will be very different from four dimensions. Twistor space of anti-self-dual four manifolds already give plenty of such manifolds which may be considered as supporting evidence.

An important class of examples come from Strominger's [65] study of the heterotic string where a hermitian metric is coupled with a stable holomorphic bundle: Given a complex manifold which admits a no-where zero holomorphic three form, a balanced metric and a stable holomorphic bundle whose first Chern class is zero, and whose second Chern form is equivalent to the second Chern form of the manifold up to $\partial \bar{\partial}$ class, then we study a coupled system of elliptic equations. The system admits interesting parallel spinors and was introduced by Strominger.

Examples were constructed by Li-Yau [42] and Fu-Yau [18]. For the twistor space mentioned above, if the anticanonical divisor exists as an effective divisor with normal crossings, we can form a branched cover of the complex manifold to kill the canonical divisor. The resulting manifold should satisfy the conditions mentioned above. Hence we expect a close relation between anti-self-dual four dimensional manifolds with such six dimensional manifolds

\section{Metric Cobordism}

The famous works by physicists on the holographic principle [44] gave the AdS/CFT correspondence, which says that if an Einstein manifold which is asymptotic to the hyperbolic metric with conformal boundary given by another manifold $M$ with positive scalar curvature, the quantum gauge theory on the boundary is isomorphic to the quantum theory of gravity in the bulk.

It opens up many interesting questions for geometry. Which compact manifold with positive scalar curvature can be written as the conformal 
boundary of hyperbolic Einstein manifolds? See [68]. How do we describe the moduli space of such manifolds?

A remarkable holographic principle appears in classical general relativity that is related to the positive mass conjecture and quasi-local mass. For a space-like two dimensional surface in a spacetime which has space-like mean curvature vector and satisfies the local energy condition, a suitably defined quasilocal mass, which only depends on the boundary data attached to the two dimensional surface, vanishes only if it bounds a space-like three dimensional hypersurface that can be embedded into the Minkowski spacetime.

All these theories indicate that there should be interesting metric discussion on cobordism theory. Many people, including Hopkins-Singer [33], Dan Freed [16], Simons-Sullivan [62] and others have studied K-theory with connections. Such a theory should be compatible with metric cobordism and the AdS/CFT correspondence. A full understanding of this will be important for the future of geometry.

\section{Affine Metrics}

This problem arose in studying the construction of Calabi -Yau metrics. Suppose we have a compact manifold with a codimensional two subcomplex $C$ such that outside $C$, there is an affine structure which means that it is covered by coordinate charts whose coordinate transformations are affine transformations with determinant equal to one. The affine structure has natural monodromy group around $C$. Suppose on each chart, we have a convex function whose Hessian defines a global metric on the complement of $C$.

We are interested in classifying those metrics, which Cheng and I [12] called affine Kähler metric, whose volume form is the given volume form attached to the affine structure. The moduli of the affine structure and the metrics and also the possible structure of $C$ and the monodromy group around $C$ needs to be classified.

This is a subject which is much related to the subject of affine spheres in the theory of affine geometry. For two dimensional affine spheres in three dimensional affine space, some of them are invariant under the affine group and the quotient is a nice Riemann surface equipped with a holomorphic cubic form. It is not clear whether there is a higher dimensional analogue of such a construction.

In this regard, there is a study of Einstein's equation in $2+1$ formulation by studying the constraint equations on Riemann surfaces. Since it satisfies the Einstein equation, the spacetime that evolves is flat due to dimensional consideration. The universal cover of the surface can be embedded into Minkowski spacetime as a hypersurface. The movement of the Riemann surface due to the Einstein flow is not trivial. It moves the conformal structure along some direction in the Teichmüller space. Moncrief and I tried to prove that this movement will deform the conformal structure to the Thurston boundary. 


\section{Isometric Embeddings}

The question of local or global isometric embeddings of a Riemannian manifold to Euclidean space is still far away from being answered. Most of the interesting progress has been made for two dimensional surfaces, where existence and uniqueness are relatively easier to understand. The uniqueness is a big mess in higher dimension because the optimal embedding dimension is $n(n+1) / 2$ and this makes the codimension too high for uniqueness, as long as the present technology is concerned. When $n>3$, the local embedding problem is a hyperbolic system. But little is known about them.

Perhaps we can try some more restricted question:

Assume a closed manifold can be conformally embedded into Euclidean space as a hypersurface, can it be deformed to be an isometric embedding? And can we prove it is unique? Perhaps some assumption such as the positivity of scalar curvature or other stronger curvature assumption should be made?

Isometric embedding into Lorentizian space time can be easier and interesting due to applications for general relativity. In many cases, we are interested to isometrically embed a spacelike hypersurface into Minkowski spacetime or some other homogenous spacetime as codimensional two spacelike hypersurface.

In the works that I did with Mu-Tao Wang, we can minimize energy among such embeddings to find some canonical one. And they can exhibit beautiful properties. Problems in higher dimensions need to be overcome.

A simple question that I encountered about thirty years ago is the following:

Is it true that a complete manifold with Ricci curvature bounded from below can be isometrically embedded into Euclidean space with bounded mean curvature?

The problem of isometric deformation of manifolds is still far from being solved. I remember I gave lectures on this subject in Berkeley in 1977. Besides the surfaces with nonnegative curvature and some surfaces with rotational symmetry, really not much is known about the problem. But even for surfaces of rotation, it is interesting enough. For in some very special cases, the problem is related to the problem of the spectrum of operators.

\section{Minimal Submanifolds}

The subject of minimal submanifolds should be considered as one of the major foundation of non-linear elliptic theory, where major techniques were developed.

In two dimensions, conformal geometry brought in beautiful technology. I am glad to see great developments led by Meeks [46], Colding and Minicozzi [13], Hoffman [32] and Rosenberg [49], and others. Under their hands, we see an almost complete picture of complete minimal surface theory in Euclidean space. 
There are still open problems that need to be solved though. A notable one is what some people call the Embedded Calabi-Yau Problem. The major contributors are Meeks [47, 48], Martin [45], Nadirashvilli [53], Perez and Ros. It says that for a surface to admit a complete bounded minimal embedding into the Euclidean three space, it is necessary and sufficient that every end of it has infinite genus.

Also, much more need to be said about minimal surfaces with constant mean curvature in other simply connected homogeneous three manifolds. For example, it will be useful to know whether there are only a finite number of closed embedded minimal surfaces with a fixed genus in the three sphere. (Due to the work of Choi-Schoen [9], we know the space is compact. The question is whether a continuous family of minimal surfaces exists or not.) I believe that it may be finite. And in that case, what is the number of such embedded minimal surfaces for each genus. It looks like the spectrum of Laplacian of these surfaces is very special. But concrete computations are needed before one can make any sensible statements.

There are plenty of works on minimal surfaces in general three dimensional manifolds. The most notable development starts from Morrey [52], and then Sacks-Uhlenbeck [58]. The bubbling of spheres and its applications to geometry are tremendous. The argument was made precise in the proof by Siu-Yau [63] of the Frankel conjecture. The proof of the topological theorem of Micallef-Moore [50] also depends on this argument. The developments of $J$-holomorphic curves in symplectic geometry owed a great deal to the work of Sacks-Uhlenbeck beyond what some authors would like to acknowledge.

There is also the great achievement of Simon-Smith [61] on the existence of embedded spheres with index less than one. Jost [36] generalized the work of Simon-Smith to the existence of two spheres in any manifold diffeomorphic to the three sphere. Colding-Minicozzi [14] studied such minimal surfaces and gave a very important application to the extinction time theorem for Hamilton's flow on simply connected three manifolds.

There is still much to be learned about how minimal surfaces are created and disappear when the metric of the three manifold changes. This is related to the appearance of black holes in general relativity.

As for higher dimensional minimal submanifolds, regularity theory has been a central problem, mostly related to geometric measure theory.

For minimal submanifolds of manifolds with special holonomy group, there are many special submanifolds that are of great interest. They are part of the calibrated submanifolds discussed by Harvey-Lawson. In many ways, they are analogues of complex submanifolds of Kähler manifolds where the restrictions of the Kähler form raised to a suitable power becomes the volume form. For manifolds with special holonomy groups, there are such forms that are parallel. The most notable is the holomorphic $n$-form of the Calabi-Yau manifolds. They define a special class of submanifolds that minimize volume in their homology class. 
Such calibrated submanifolds were rediscovered by Becker-BeckerStrominger $[\mathbf{2}]$ in their study of cycles that preserve some supersymmetry of the ambient manifolds. They are special cases of branes in string theory. As such, they play important roles in the consideration of supersymmetry, which gives beautiful duality with holomorphic bundles. Many analogues with holomorphic bundles can be drawn.

Richard Thomas and I [67] looked into various properties of such cycles. We conjectured that classes of lagrangian cycles with zero Maslov index should admit special lagrangian cycles and that they can be obtained by the mean curvature flow where long time existence should hold. And if some stability condition holds, it will converge to special lagrangian cycles. It is important to check that special lagrangian cycles automatically satisfies such stability conditions. The mirror symmetry between these cycles with Hermitian-Yang-Mills bundles is really fascinating.

An important question that I raised many years ago, is the mirror of the Hodge conjecture. The question here is that every middle dimensional cycles of the odd dimensional Calabi-Yau manifold should be written as a (rational) linear combination of special lagrangian cycles. Note that special lagrangian cycles mean cycles that can be calibrated by possibly different choices of $(n, 0)$-forms with norm one, and also the coefficients can have different signs.

If we insist that the coefficients be positive, then there is an interesting question: When will a homology class be representable by an irreducible special lagrangian cycle?

Once we have obtained a special lagrangian cycle, we would like to know its topology. For a Calabi-Yau manifold, what kind of topology can a special lagrangian cycle admit? Can a real hyperbolic manifold occur? When will it be a torus? When it is a torus, what is its deformation space? Can we fill in the Calabi-Yau manifold with special lagrangian tori so that the moduli space can be identified with some three-manifold with an affine structure that has singularity along some codimensional two set. This is related to what is called the Strominger-Yau-Zaslow [66] fibration and the mirror conjecture.

\section{References}

[1] S. Bando, On the classification of three-dimensional compact Kaehler manifolds of nonnegative bisectional curvature. J. Differential Geom. 19(1984), no. 2, 283-297.

[2] K. Becker, M. Becker, Melanie and A. Strominger, Fivebranes, membranes and nonperturbative string theory. Nuclear Phys. B 456 (1995), no. 1-2, 130-152.

[3] S. Brendle and R. Schoen, Manifolds with 1/4-pinched curvature are space forms. J. Amer. Math. Soc. 22(2009), no. 2, 287-307.

[4] C. P. Boyer and K. Galicki, Sasakian geometry, Oxford Mathematical Monographs, Oxford University Press (2007).

[5] H. D. Cao, Deformation of Kähler metrics to Kähler-Einstein metrics on compact Kähler manifolds. Invent. Math. 81(1985), no. 2, 359-372. 
[6] H. D. Cao, On Harnack inequalities for the Kähler-Ricci flow. Invent. Math. 109(1992), 247-263.

[7] H. D. Cao, Limits of solutions to the Kähler-Ricci flow. J. Differential Geom. 45(1997), no. 2, 257-272.

[8] H.D. Cao and B. Chow, Compact Kähler manifolds with nonnegative curvature operator. Invent. Math. 83(1986), 553-556.

[9] H. I. Choi and R. Schoen, The space of minimal embeddings of a surface into a threedimensional manifold of positive Ricci curvature. Invent. Math. 81(1985), no. 3, 387-394.

[10] A. Chau and L.-F. Tam, On the complex structure of Kähler manifolds with nonnegative curvature. arXiv:math.DG/0504422.

[11] J. Cheeger and D. Gromoll, On the structure of complete manifolds of nonnegative curvature. Ann. of Math., 96 (1978) no. 3, 413-443.

[12] S. Y. Cheng and S.-Y. Yau, The real Monge-Ampère equation and affine flat structures, in Proceedings of the 1980 Beijing Symposium on Differential Geometry and Differential Equations, Science Press, Beijing, China (1982), Gordon and Breach Science Publishing Company, New York , pp. 339-370.

[13] T. H. Colding and W. P. Minicozzi II The space of embedded minimal surfaces of fixed genus in a 3-manifold. Ann. of Math. (2) 160(2004), I., 27-68; II., 69-92; III., 523-572; IV., 573-615.

[14] T. H. Colding and W. P. Minicozzi II Estimates for the extinction time for the Ricci flow on certain 3-manifolds and a question of Perelman. J. Amer. Math. Soc., 18 (2005) no. 3, 561-569.

[15] S. K. Donaldson, Scalar curvature and projective embeddings. I. J. Differential Geom. 59(2001), no. 3, 479-522.

[16] D. S. Freed, Pions and generalized cohomology. J. Differential Geom. 80(2008), 45-77.

[17] M. H. Freedman and F. Quinn, Topology of 4-manifolds, Princeton Mathematical Series, 39., Princeton University Press, Princeton, NJ, 1990.

[18] J. X. Fu and S.-T. Yau, The theory of superstring with flux on non-Kahler manifolds and the complex Monge-Ampere equation. J. Differential Geom. 78(2008), 369-428.

[19] M. E. Gage, On an area-preserving evolution equation for plane curves, pp. 51-62. in Nonlinear problems in geometry (Mobile, Ala., 1985), edited by D. M. DeTurck, Contemporary Mathematics 51, Amer. Math. Soc., Providence, 1986.

[20] M. E. Gage and R. S. Hamilton, The heat equation shrinking convex plane curves. J. Differential Geom., 23 (1986), 69-96.

[21] G. W. Gibbons, S. W. Hawking, and M. J. Perry, Path integrals and the indefiniteness of the gravitational action. Nuclear Phys. B, 138:1 (1978), 141-150.

[22] A. Gray, Nearly Kahler manifolds. J. Differential Geom. 4 (1970), 283-309

[23] M. A. Grayson, The heat equation shrinks embedded plane curves to round points. J. Differential Geom., 26 (1987), 285-314.

[24] M. Gromov, Curvature, diameter and Betti numbers. Comment. Math. Helv. 56 (1981), 179-195.

[25] M. Gromov and H. B. Lawson, The classification of simply connected manifolds of positive scalar curvature. Ann. of Math.,111 (3) (1980), 423-434.

[26] R. S. Hamilton, Three-manifolds with positive Ricci curvature. J. Differential Geom. 17(1982), no. 2, 255-306.

[27] R. S. Hamilton, The Harnack estimate for the Ricci flow. J. Differential Geom. 37(1993), no. 1, 225-243.

[28] R. S. Hamilton, The formation of singularities in the Ricci flow. Surveys in differential geometry, Vol. II (Cambridge, MA, 1993), 7-136, Internat. Press, Cambridge, MA, 1995. 
[29] R. S. Hamilton, Four-manifolds with positive isotropic curvature. Comm. Anal. Geom. 5(1997), no. 1, 1-92.

[30] R. S. Hamilton, Non-singular solutions of the Ricci flow on three-manifolds. Comm. Anal. Geom. 7(1999), no. 4, 695-729.

[31] N. J. Hitchin, Kählerian twistor spaces. Proc. London Math. Soc. 43 (1981), 133-150.

[32] D. Hoffman, Computing minimal surfaces. Global theory of minimal surfaces, 259-282, Clay Math. Proc., 2, Amer. Math. Soc., Providence, RI, 2005.

[33] M. J. Hopkins and I. M. Singer, Quadratic functions in Geometry, Topology, and M-theory. J. Differential Geom. 70(2005) 329-452.

[34] G. Huisken, Flow by mean curvature of convex surfaces into spheres. J. Differential Geom. 20(1984), no. 1, 237-266.

[35] G. Huisken, Contracting convex hypersurfaces in Riemannian manifolds by their mean curvature. Invent. Math. 84(1986), no. 3, 463-480.

[36] J. Jost, Embedded minimal surfaces in manifolds diffeomorphic to the threedimensional ball or sphere. J. Differential Geom. 30(1989), no. 2, 555-577.

[37] D. D. Joyce, Compact 8-manifolds with holonomy Spin(7). Invent. Math. 123(1996), no. $3,507-552$.

[38] D. D. Joyce, Compact Riemannian 7-manifolds with holonomy $G_{2}$. I, II. J. Differential Geom. 43(1996), no. 2, 291-328, 329-375.

[39] D. Joyce, A new construction of compact 8-manifolds with holonomy Spin(7). J. Differential Geom. 53(1999), no. 1, 89-130.

[40] D. Joyce, Compact Riemannian manifolds with special holonomy, 436 pages, Oxford University Press, 2000.

[41] H. B. Lawson Jr. and S.-T. Yau, Scalar curvature, non-abelian group actions, and the degree of symmetry of exotic spheres. Comment. Math. Helv. 49(1974), 232-244.

[42] J. Li and S.-T. Yau, The Existence of Supersymmetric String Theory with Torsion. J. Differential Geom. 70(2005), 143-181.

[43] P. Li and S.-T. Yau, On the parabolic kernel of the Schrödinger operator. Acta Math. 156(1986), no. 3-4, 153-201.

[44] J. M. Maldacena, The Large N limit of superconformal field theories and supergravity. Adv. Theor. Math. Phys. 2(1998), 231-252.

[45] F. Martin and S. Morales, Complete proper minimal surfaces in convex bodies of $\mathbb{R}^{3}$. Duke Math. J. 128 (2005), no. 3, 559-593.

[46] W. H. Meeks, III, Applications of minimal surfaces to the topology of three-manifolds. Current developments in mathematics, 2004, 95-109, Int. Press, Somerville, MA, 2006.

[47] W. H. Meeks, III, J. Perez and A. Ros, The geometry of minimal surfaces of finite genus. I. Curvature estimates and quasiperiodicity. J. Differential Geom. 66(2004), $1-45$.

[48] W. H. Meeks, III, J. Perez and A. Ros, Liouville-type properties for embedded minimal surfaces. Comm. Anal. Geom. 14(2006), no. 4, 703-723.

[49] W. H. Meeks, III and H. Rosenberg, The minimal lamination closure theorem. Duke Math. J. 133(2006), 467-497.

[50] M. Micallef and J.D. Moore, Minimal two-spheres and the topology of manifolds with positive curvature on totally isotropic two-planes. Ann. of Math. (2) 27(1988), 199-227.

[51] N. Mok, The uniformization theorem for compact Kähler manifolds of nonnegative holomorphic bisectional curvature. J. Differential Geom. 27(1988), no. 2, 179-214.

[52] C. B. Morrey, The problem of Plateau in a Riemannian manifold. Ann. of Math. 49(1948), 807-851.

[53] N. Nadirashvili, Hadamard's and Calabi-Yau's conjectures on negatively curved and minimal surfaces. Invent. Math. 126 (1996) 457-465. 
[54] G. Perelman, Proof of the soul conjecture of Cheeger and Gromoll. J. Differential Geom 40(1994), no. 1, 209-212.

[55] G. Perelman, The entropy formula for the Ricci flow and its geometric applications, arXiv:math.DG/0211159.

[56] G. Perelman, Ricci flow with surgery on three-manifolds, arXiv:math/0303109.

[57] G. Perelman, Finite extinction time for the solutions to the Ricci flow on certain three-manifolds, arXiv:math.DG/0307245.

[58] J. Sacks and K. Uhlenbeck, The existence of minimal immersions of 2-spheres. Ann. of Math. (2) 113(1981), no. 1, 1-24.

[59] R. Schoen and S.-T. Yau, On the structure of manifolds with positive scalar curvature, Manuscripta Math. 28 (1979), 159-183.

[60] R. Schoen and S.-T. Yau, Complete three-dimensional manifolds with positive Ricci curvature and scalar curvature. Seminar on Differential Geometry, pp. 209-228, Ann. of Math. Stud., 102, Princeton Univ. Press, Princeton, N.J., 1982.

[61] L. Simon and F. Smith, On the existence of embedded minimal 2-spheres in the 3-sphere, endowed with an arbitrary metric, unpublished.

[62] J. Simons and D. Sullivan, Structured vector bundles define differential K-theory. arXiv:0810.4935.

[63] Y. T. Siu and S.-T. Yau, Compact Kähler manifolds of positive bisectional curvature. Invent. Math. 59(1980), no. 2, 189-204.

[64] S. Stolz, Simply connected manifolds of positive scalar curvature. Ann. of Math. 136 (3) (1992), 511-540.

[65] A. Strominger, Superstrings with torsion. Nuclear Phys. B 274(1986), no. 2, 253284.

[66] A. Strominger, S.-T. Yau and E. Zaslow, Mirror symmetry is T-duality. Nuclear Phys. B 479(1996), no. 1-2, 243-259.

[67] R. P. Thomas and S.-T. Yau, Special Lagrangians, stable bundles and mean curvature flow. (English. English summary) Comm. Anal. Geom. 10(2002), no. 5, 1075-1113.

[68] E. Witten and S.-T. Yau, Connectedness of the boundary in the AdS/CFT correspondence. Adv. Theor. Math. Phys. 3(1999), no. 6, 1635-1655 (2000).

[69] S.-T. Yau, A review of complex differential geometry. Proc. Symp. Pur Math. 52(1991), 619-625.

[70] S.-T. Yau, Open problems in Geometry. Proc. Symp. Pure Math. 54(1993) 1-28.

[71] S.-T. Yau, Métriques de Kähler-Einstein sur les variétés ouvertes; Premiére Classe de Chern et courbure de Ricci: Preuve de la conjecture de Calabi. Séminaire Palaiseau, Astérisque, 58 (1978), 163-167.

[72] Shiu Yuen Cheng and Shing-Tung Yau, On the existence of a complete Kähler metric on noncompact complex manifolds and the regularity of Fefferman's equation. Comm. Pure Appl. Math. 33 (1980), no. 4, 507-544.

[73] Shiu Yuen Cheng and Shing-Tung Yau, Inequality between Chern numbers of singular Kähler surfaces and characterization of orbit space of discrete group of $S U(2,1)$; Complex differential geometry and nonlinear differential equations. (Brunswick, Maine, 1984), 31-44, Contemp. Math., 49, Amer. Math. Soc., Providence, RI, 1986.

[74] Gang Tian and Shing-Tung Yau, Complete Kähler manifolds with zero Ricci curvature, I. J. Amer. Math. Soc. 3 (1990), no. 3, 579-609.

[75] Gang Tian and Shing-Tung Yau, Complete Kähler manifolds with zero Ricci curvature, II. Invent. Math. 106 (1991), no. 1, 27-60.

Department of Mathematics, Harvard University, One Oxford Street, Cambridge, MA 02138, USA

E-mail address: yau@math.harvard.edu 
